\title{
Wa Ve phenomena

\section{Existence of cylindrically symmetric ground states to a nonlinear curl-curl equation with non-constant coefficients}

Andreas Hirsch, Wolfgang Reichel

CRC Preprint 2016/14, June 2016

\section{KARLSRUHE INSTITUTE OF TECHNOLOGY}

\section{CRC 1173}

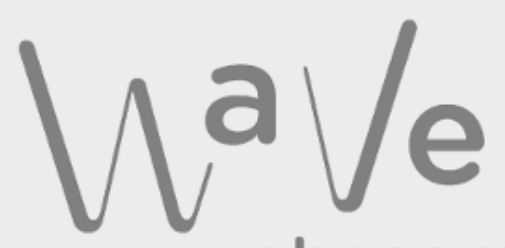

phenomena 


\section{Participating universities}

EBERHARD KARLS

UNIVERSITATT TUBINGEN

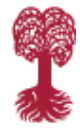

Funded by

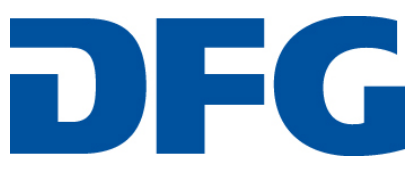

ISSN 2365-662X 


\title{
EXISTENCE OF CYLINDRICALLY SYMMETRIC GROUND STATES TO A NONLINEAR CURL-CURL EQUATION WITH NON-CONSTANT COEFFICIENTS
}

\author{
ANDREAS HIRSCH AND WOLFGANG REICHEL
}

\begin{abstract}
Aвstract. We consider the nonlinear curl-curl problem $\nabla \times \nabla \times U+V(x) U=f\left(x,|U|^{2}\right) U$ in $\mathbb{R}^{3}$ related to the nonlinear Maxwell equations with Kerr-type nonlinear material laws. We prove the existence of a symmetric ground-state type solution for a bounded, cylindrically symmetric coefficient $V$ and subcritical cylindrically symmetric nonlinearity $f$. The new existence result extends the class of problems for which ground-state type solutions are known. It is based on compactness properties of symmetric functions [11, 12], new rearrangement type inequalities from [6] and the recent extension of the Nehari-manifold technique from [18].
\end{abstract}

\section{INTRODUCTION}

We consider the system

$$
\nabla \times \nabla \times U+V(x) U=f\left(x,|U|^{2}\right) U \text { in } \mathbb{R}^{3}
$$

where $V \in L^{\infty}\left(\mathbb{R}^{3}\right)$ and $f: \mathbb{R}^{3} \times[0, \infty) \rightarrow[0, \infty)$ is a non-negative Carathéodory function growing at infinity with a power at most $\frac{p-1}{2}$ for $p \in(1,5)$. The particular feature of (1.1) is the curl-curl operator. It arises in specific models for standing waves in Maxwell's equations with Kerr-type nonlinear material laws where $f\left(x,|U|^{2}\right) U=\Gamma(x)|U|^{2} U$. For a detailed physical motivation of (1.1) see [2].

We look for $\mathbb{R}^{3}$-valued weak solutions $U$ in a cone $K_{4,1}$ of functions with suitable symmetries and $U \in L^{2}\left(\mathbb{R}^{3}\right) \cap L^{p+1}\left(\mathbb{R}^{3}\right), \nabla \times U \in L^{2}\left(\mathbb{R}^{3}\right)$. The condition that 0 lies below the spectrum of curl curl $+V(x)$ allows us to find ground-state type critical points of a functional $J(u)=\frac{1}{2}\|u\|^{2}-I(u)$, cf. (1.4), restricted to the so-called Nehari-manifold. The basic idea of applying symmetrizations to minimizing sequences on the Nehari-manifold goes back to Stuart [17] in the context of the stationary nonlinear Schrödinger equation. Compared to [17] the assumptions on the nonlinearity $f$ can be substantially weakened beyond the classical Ambrosetti-Rabinowitz condition. This is based on three important ingredients:

- the recent extension of the Nehari-manifold method due to Szulkin and Weth [18],

- the weak sequential continuity of functionals $I(u)$ and $I^{\prime}(u)[u]$ on $K_{4,1}$ due to compactness properties of symmetric functions by Lions [11, 12],

- new rearrangement inequalities for general nonlinearities due to Brock [6].

Using the combination of these ingredients our main result of Theorem 1 substantially extends the know results on the existence of ground-state type solutions for (1.1).

Benci, Fortunato [5] and Azzollini, Benci, D’Aprile, Fortunato in [1] were among the first to consider the constant coefficient case of (1.1) with $V \equiv 0$. Their method was based on cylindrical symmetries of the vector-fields $U$, cf. [8] for a different class of symmetries. The case where

Date: June 15, 2016.

2000 Mathematics Subject Classification. Primary: 35J20, 58E15; Secondary: 47J30, 35Q60.

Key words and phrases. curl-curl problem, nonlinear elliptic equations, cylindrical symmetry, variational methods. 
$f\left(x,|U|^{2}\right) U=\Gamma(x)|U|^{p-1} U$ with periodic coefficients $V$ and $\Gamma$ has been treated in [2]. In [14] Mederski considered (1.1) where $f\left(x,|U|^{2}\right) U$ is replaced by, e.g., $\Gamma(x) g(U)$ with $\Gamma>0$ periodic and bounded, $V \leq 0, V \in L^{\frac{p+1}{p-1}}\left(\mathbb{R}^{3}\right) \cap L^{\frac{q+1}{q-1}}\left(\mathbb{R}^{3}\right)$ and $g(U) \sim|u|^{p-1} U$ if $|U| \gg 1$ and $g(U) \sim|U|^{q-1} u$ if $|U| \ll 1$ for $1<p<5<q$. A remarkable feature of Mederski's work is that (1.1) can be treated without assuming special symmetries of the field $U$. The nonlinear curl-curl problem on bounded domains with the boundary condition $v \times U=0$ has been elaborated in [3, 4].

An important feature of [1] is the use of cylindrically symmetric ansatz functions for $U$. Here we make a slightly different ansatz of the form

$$
U(x)=u(r, z)\left(\begin{array}{c}
-x_{2} \\
x_{1} \\
0
\end{array}\right) \text { where } r=\sqrt{x_{1}^{2}+x_{2}^{2}}, z=x_{3} .
$$

Moreover, we assume cylindrically symmetric coefficients $V(x)=V(r, z), f\left(x,|U|^{2}\right)=f\left(r, z,|U|^{2}\right)$. For $U$ of the form (1.2) we see that $\operatorname{div} U=0$, and hence (1.1) reduces to the scalar equation

$$
-\frac{1}{r^{3}} \frac{\partial}{\partial r}\left(r^{3} \frac{\partial u}{\partial r}\right)-\frac{\partial^{2} u}{\partial z^{2}}+V(r, z) u=f\left(r, z, r^{2} u^{2}\right) u \quad \text { for } \quad(r, z) \in \Omega:=(0, \infty) \times \mathbb{R} .
$$

It turns out that a suitable space to consider 1.3 is given by

$$
\begin{aligned}
H_{\mathrm{cyl}}^{1}\left(r^{3} d r d z\right) & :=\left\{v:(0, \infty) \times \mathbb{R} \rightarrow \mathbb{R}: v, \frac{\partial v}{\partial r}, \frac{\partial v}{\partial z} \in L_{\mathrm{cyl}}^{2}\left(r^{3} d r d z\right)\right\}, \\
L_{\mathrm{cyl}}^{2}\left(r^{3} d r d z\right) & :=\left\{v:(0, \infty) \times \mathbb{R} \rightarrow \mathbb{R}: \int_{\Omega} v(r, z)^{2} r^{3} d(r, z)<\infty\right\},
\end{aligned}
$$

cf. Section 2 for more details on these spaces. Weak solutions of (1.3) arise as critical points of the functional

$$
J(u)=\frac{1}{2} \int_{\Omega}\left(\left|\nabla_{r, z} u\right|^{2}+V(r, z) u^{2}\right) r^{3} d(r, z)-\int_{\Omega} \frac{1}{2 r^{2}} F\left(r, z, r^{2} u^{2}\right) r^{3} d(r, z), u \in H_{\mathrm{cyl}}^{1}\left(r^{3} d r d z\right),
$$

where $F(r, z, t):=\int_{0}^{t} f(r, z, s) d s$ and $\nabla_{r, z}:=\left(\frac{\partial}{\partial r}, \frac{\partial}{\partial z}\right)$. A ground state $u$ of (1.3) is defined as a weak solution of (1.3) in the Nehari-manifold

$$
M:=\left\{v \in H_{\mathrm{cyl}}^{1}\left(r^{3} d r d z\right) \backslash\{0\}: \int_{\Omega}\left(\left|\nabla_{r, z} v\right|^{2}+V(r, z) v^{2}\right) r^{3} d(r, z)=\int_{\Omega} f\left(r, z, r^{2} v^{2}\right) v^{2} r^{3} d(r, z)\right\}
$$

such that

$$
J(u)=\inf _{v \in M} J(v),
$$

see the classical papers [15], [16]. We find ground states of (1.3) under additional assumptions on $V$ and $f$. To state these assumptions we need the notion of Steiner-symmetrization, cf. Chapter 3 in [10]. The Steiner-symmetrization (also called symmetric-deacreasing rearrangement) of a cylindrical function $g=g(r, z)$ with respect to $z$ is denoted by $g^{\star}$. We say that $g$ is Steiner-symmetric if $g$ coincides with its Steiner-symmetrization with respect to $z$, keeping the $r$-variable fixed. A function $h \in L^{\infty}(\Omega)$ is reversed Steiner-symmetric if (ess sup $h-h)^{\star}=\operatorname{ess} \sup h-h$ holds true.

Now we can state our assumptions on $f$.

(i) $f: \Omega \times[0, \infty) \rightarrow \mathbb{R}$ is a Carathéodory function with $0 \leq f(r, z, s) \leq c\left(1+s^{\frac{p-1}{2}}\right)$ for some $c>0$ and $p \in(1,5)$,

(ii) $f(r, z, s)=o(1)$ as $s \rightarrow 0$ uniformly in $r, z \in[0, \infty) \times \mathbb{R}$,

(iii) $f(r, z, s)$ strictly increasing in $s \in[0, \infty)$, 
(iv) $\frac{F(r, z), s}{s} \rightarrow \infty$ as $s \rightarrow \infty$ uniformly in $r, z \in[0, \infty) \times \mathbb{R}$,

(v) for all $r \in[0, \infty), s \geq 0$ and $\sigma>0$ the function

$$
\varphi_{\sigma}(r, z, s):=f\left(r, z,(s+\sigma)^{2}\right)(s+\sigma)^{2}-f\left(r, z, s^{2}\right) s^{2}
$$

is symmetrically nonincreasing in $z$.

Conditions (ii)-(iv) are inspired by the work of Szulkin and Weth [18]. Namely, if we translate (ii)(iv) into conditions for $\tilde{f}(r, z, s):=f\left(r, z, r^{2} s^{2}\right) s$ then they become identical to (ii)-(iv) of Theorem 20 from [18]. Condition (v) is used to prove the rearrangement inequality of Lemma 9 and it is due to Brock [6].

Next we state our main result.

Theorem 1. Let $V \in L^{\infty}(\Omega)$ be reversed Steiner-symmetric such that the map

$$
\|\cdot\|: H_{\mathrm{cyl}}^{1}\left(r^{3} d r d z\right) \rightarrow \mathbb{R} ; u \mapsto\left(\int_{\Omega}\left(\left|\nabla_{r, z} u\right|^{2}+V(r, z) u^{2}\right) r^{3} d(r, z)\right)^{\frac{1}{2}}
$$

is an equivalent norm to $\|\cdot\|_{H_{\mathrm{cyl}}^{1}\left(r^{3} d r d z\right)}$. Additionally, let $f$ satsify the assumptions $(i)-(v)$. Then (1.3) has a ground state $u \in H_{\mathrm{cyl}}^{1}\left(r^{3} d r d z\right)$ which is symmetric about $\{z=0\}$.

Remarks: (1) The assumption of norm-equivalence is for instance satisfied if $V \geq 0$ and $\inf _{B_{R}^{c}} V>0$ for some $R>0$, where $B_{R}^{c}:=\left\{(r, z) \in \Omega: r^{2}+z^{2}>R^{2}\right\}$. For the reader's convenience the proof based on Poincaré's inequality is given in the Appendix. Since Poincaré's inequality is applicable for domains bounded in one direction we can weaken $\inf _{B_{R}^{c}} V>0$ to $\inf _{S^{c}} V>0$ for strips $S=[0, \infty) \times[0, \rho]$ with $\rho>0$ or $S=\left[r_{0}, r_{1}\right] \times[0, \infty)$ with $0 \leq r_{0}<r_{1}<\infty$.

(2) The conditions on $f$ are satisfied if for instance $f(r, z, s)=\Gamma(r, z)|s|^{\frac{p-2}{2}} s$ where $\Gamma \in L^{\infty}(\Omega)$ is Steiner-symmetric, ess $\inf _{\Omega} \Gamma>0$ and $p \in(1,5)$. This choice of $f$ corresponds to the equation $\nabla \times \nabla \times U+V(r, z) U=\Gamma(r, z)|U|^{p-1} U$ in $\mathbb{R}^{3}$. Another possible choice is $f(r, z, s)=\Gamma(r, z) \log (1+s)$

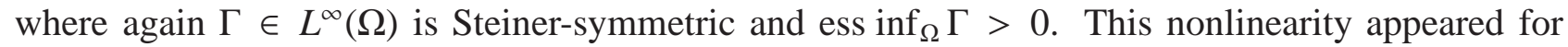
instance in [13] and it does not satisfy the classical Ambrosetti-Rabinowitz condition.

The paper is structured as follows: In Section 2 we give details on the variational formulation of problem (1.3) and prove pointwise decay estimates of Steiner-symmetric functions in $H_{\text {cyl }}^{1}\left(r^{3} d r d z\right)$. In Section 3 we give the proof of Theorem 11 and in the Appendix we show an example for the potential $V$ satisfying the equivalent-norm-assumption of Theorem 1 .

\section{VARIATIONAL FORMULATION, DECAY ESTIMATES, REARRANGEMENTS}

Let us consider some properties of the space $H_{\text {cyl }}^{1}\left(r^{3} d r d z\right)$. First, for $U$ of the form (1.2) we have that $U \in H^{1}\left(\mathbb{R}^{3}\right)$ if and only if $u \in H_{\text {cyl }}^{1}\left(r^{3} d r d z\right)$. A norm on $H_{\text {cyl }}^{1}\left(r^{3} d r d z\right)$ is given by

$$
\|u\|_{H_{\mathrm{cyl}}^{1}\left(r^{3} d r d z\right)}:=\left(\int_{\Omega}\left(\left|\nabla_{r, z} u\right|^{2}+u^{2}\right) r^{3} d(r, z)\right)^{\frac{1}{2}} .
$$

Notice that the space $H_{\text {cyl }}^{1}\left(r^{3} d r d z\right)$ behaves like a Sobolev-space in dimension 5. Next we show a useful embedding property. For this we need the following Sobolev and Lebesgue spaces in dimension 3 together with their canonical norms:

$$
H_{\mathrm{cyl}}^{1}(r d r d z):=\left\{v:(0, \infty) \times \mathbb{R} \rightarrow \mathbb{R}: v, \frac{\partial v}{\partial r}, \frac{\partial v}{\partial z} \in L_{\mathrm{cyl}}^{2}(r d r d z)\right\},
$$




$$
L_{\text {cyl }}^{q}(r d r d z):=\left\{v:(0, \infty) \times \mathbb{R} \rightarrow \mathbb{R}: \int_{\Omega}|v(r, z)|^{q} r d(r, z)<\infty\right\} \text { for } q \in[1, \infty) .
$$

Lemma 2. For $u \in H_{\mathrm{cyl}}^{1}\left(r^{3} d r d z\right)$ Hardy's inequality holds

$$
\int_{\Omega} \frac{u^{2}}{r^{2}} r^{3} d(r, z) \leq C_{H} \int_{\Omega}\left(\left(\frac{\partial u}{\partial r}\right)^{2}+\left(\frac{\partial u}{\partial z}\right)^{2}\right) r^{3} d(r, z)
$$

Moreover, if $u \in H_{\mathrm{cyl}}^{1}\left(r^{3} d r d z\right)$ then $r u \in H_{\mathrm{cyl}}^{1}(r d r d z)$ and there is a constant $C>0$ such that for $2 \leq q \leq 6$

$$
\|r u\|_{H_{\mathrm{cyl}}^{1}(r d r d z)},\|r u\|_{L_{\mathrm{cyl}}^{q}(r d r d z)} \leq C\|u\|_{H_{\mathrm{cyl}}^{1}\left(r^{3} d r d z\right)}
$$

Proof. Hardy's inequality (2.1) is given in Lemma 9 (i) in [2]. For $u \in H_{\text {cyl }}^{1}\left(r^{3} d r d z\right)$ we have $r u, \frac{\partial}{\partial z}(r u)$, $r \frac{\partial u}{\partial r} \in L_{\text {cyl }}^{2}(r d r d z)$ and by (2.1) also $u \in L_{\text {cyl }}^{2}(r d r d z)$. Since $\frac{\partial}{\partial r}(r u)=r \frac{\partial u}{\partial r}+u$ we conclude altogether $r u \in H_{\text {cyl }}^{1}(r d r d z)$. By the Sobolev embedding in three dimensions this implies $r u \in L^{q}(r d r d z)$ for $q \in[2,6]$ and (2.1) yields

$$
\begin{aligned}
\|r u\|_{H_{\mathrm{cyl}}^{1}(r d r d z)}^{2} & =\int_{\Omega}\left(\left|\nabla_{r, z}(r u)\right|^{2}+r^{2} u^{2}\right) r d(r, z) \\
& \leq 2 \int_{\Omega}\left(\left(r \frac{\partial u}{\partial z}\right)^{2}+\left(r \frac{\partial u}{\partial r}\right)^{2}+u^{2}+r^{2} u^{2}\right) r d(r, z) \leq \tilde{C}\|u\|_{H_{\mathrm{cyl}}^{1}\left(r^{3} d r d z\right)}^{2} .
\end{aligned}
$$

Next we show that the functional $J$ from the introduction as well as the functional in the defintion of the Nehari-manifold are well-defined.

Lemma 3. There is a constant $C>0$ such that

$$
\int_{\Omega} f\left(r, z, r^{2} u^{2}\right) u^{2} r^{3} d r d z, \int_{\Omega} \frac{1}{2 r^{2}} F\left(r, z, r^{2} u^{2}\right) r^{3} d(r, z) \leq C\left(\|u\|_{H_{\mathrm{cyl}}^{1}\left(r^{3} d r d z\right)}^{2}+\|u\|_{H_{\mathrm{cyl}}^{1}\left(r^{3} d r d z\right)}^{p+1}\right)
$$

for all $u \in H_{\mathrm{cyl}}^{1}\left(r^{3} d r d z\right)$.

Proof. Clearly assumption (i) and (ii) show that for every $\epsilon>0$ there is $C_{\epsilon}>0$ such that

$$
0 \leq f(r, z, s) \leq \epsilon+C_{\epsilon} s^{\frac{p-1}{2}} .
$$

Hence

$$
\begin{aligned}
0 & \left.\leq f\left(r, z, r^{2} u^{2}\right) u^{2} r^{3} \leq\left(\epsilon r^{2} u^{2}+C_{\epsilon}|r u|^{p+1}\right)\right) r, \\
0 & \leq \frac{1}{2 r^{2}} F\left(r, z, r^{2} u^{2}\right) r^{3} \leq\left(\epsilon r^{2} u^{2}+\tilde{C}_{\epsilon}|r u|^{p+1}\right) r .
\end{aligned}
$$

Due to (2.2) this implies the claim.

In order to find critical points of $J$ we need uniform decay estimates of Steiner-symmetric functions in $H_{\text {cyl }}^{1}\left(r^{3} d r d z\right)$. These estimates are given in [12] in much more generality but for the sake of completeness we give them here together with the simple proof. We start with a well-known fact concerning radially symmetric functions and afterwards extend the result to cylindrically symmetric functions. Let

$$
H_{\mathrm{rad}}^{1}\left(\mathbb{R}^{n}\right):=\left\{u \in H^{1}\left(\mathbb{R}^{n}\right): u \text { is radially symmetric }\right\}
$$


Lemma 4. (see [12]) Let $n \geq 2$. Then there is a constant $C>0$ such that

$$
|u(x)| \leq C\|\nabla u\|_{L^{2}\left(\mathbb{R}^{n}\right)}^{1 / 2}\|u\|_{L^{2}\left(\mathbb{R}^{n}\right)}^{1 / 2}|x|^{-(n-1) / 2} \text { for almost all } x \in \mathbb{R}^{n} \text { and all } u \in H_{\mathrm{rad}}^{1}\left(\mathbb{R}^{n}\right) .
$$

Proof. By density it is sufficient to prove the estimate for $u \in H_{\text {rad }}^{1}\left(\mathbb{R}^{n}\right) \cap C_{c}^{\infty}\left(\mathbb{R}^{n}\right)$. Let $r:=|x|$. Then

$$
\frac{d}{d r}\left(r^{n-1}|u|^{2}\right)=(n-1) r^{n-2}|u|^{2}+r^{n-1} 2 u \frac{\partial u}{\partial r} \geq-2|u|\left|\frac{\partial u}{\partial r}\right| r^{n-1} .
$$

Integrating from $r$ to $\infty$ and expanding the domain of integration to all of $\mathbb{R}^{n}$ yields

$$
r^{n-1}|u(x)|^{2} \leq C \int_{\mathbb{R}^{n}}|u||\nabla u| d y \leq C\|\nabla u\|_{L^{2}\left(\mathbb{R}^{n}\right)}\|u\|_{L^{2}\left(\mathbb{R}^{n}\right)} .
$$

Now we give an extension of Lemma 4 to cylindrically symmetric functions which are Steinersymmetric in the non-radial component. We make use of the following notation: Let $t \in \mathbb{N}_{\geq 2}$ and $s \in \mathbb{N}$ such that $n=t+s$. We write points in $\mathbb{R}^{n}$ as $(x, y)$ with $x \in \mathbb{R}^{t}$ and $y=\left(y_{1}, \ldots, y_{s}\right) \in \mathbb{R}^{s}$. Furthermore, let

$$
K_{t, s}:=\left\{u \in H^{1}\left(\mathbb{R}^{n}\right) \text { s.t. }\left\{\begin{array}{ll}
u(\cdot, y) & \text { is a radially symmetric function for every } y \in \mathbb{R}^{s} \text { and } \\
u(x, \cdot) & \text { is Steiner-symmetric w.r.t. } y_{i}, i=1, \ldots, s, \text { for every } x \in \mathbb{R}^{t}
\end{array}\right\} .\right.
$$

In particular, if $u \in K_{t, s}$ then necessarily $u \geq 0$. In this setting we have the following extension of Lemma 4.

Lemma 5. (see [12]) There is a constant $C>0$ such that

$$
0 \leq u(x, y) \leq C\left\|\nabla_{x} u\right\|_{L^{2}\left(\mathbb{R}^{n}\right)}^{1 / 2}\|u\|_{L^{2}\left(\mathbb{R}^{n}\right)}^{1 / 2}|x|^{-(t-1) / 2}\left|y_{1} \cdots y_{s}\right|^{-1 / 2} \text { for almost all }(x, y) \in \mathbb{R}^{n} \text { and all } u \in K_{t, s} .
$$

Proof. Let $u \in K_{t, s}$ and fix $y \in \mathbb{R}^{s}$. W.1.o.g. let $y_{i}>0$ for all $i=1, \ldots, s$. We define

$$
v(x):=\int_{0}^{y_{1}} \cdots \int_{0}^{y_{s}} u(x, z) d z \text { for } x \in \mathbb{R}^{t} .
$$

By Hölder's inequality we obtain $v^{2}(x) \leq y_{1} \cdots y_{s} \int_{0}^{y_{1}} \cdots \int_{0}^{y_{s}} u^{2}(x, z) d z$, i.e.,

$$
\|v\|_{L^{2}\left(\mathbb{R}^{t}\right)} \leq\left(y_{1} \cdots y_{s}\right)^{1 / 2}\|u\|_{L^{2}\left(\mathbb{R}^{n}\right)} .
$$

In the same manner we receive

$$
\|\nabla v\|_{L^{2}\left(\mathbb{R}^{t}\right)} \leq\left(y_{1} \cdots y_{s}\right)^{1 / 2}\left\|\nabla_{x} u\right\|_{L^{2}\left(\mathbb{R}^{n}\right)} .
$$

Since $v: \mathbb{R}^{t} \rightarrow \mathbb{R}$ is radially symmetric we can apply Lemma 4 and get from (2.7) and (2.8)

$$
0 \leq v(x) \leq C\|\nabla v\|_{L^{2}\left(\mathbb{R}^{t}\right)}^{1 / 2}\|v\|_{L^{2}\left(\mathbb{R}^{t}\right)}^{1 / 2}|x|^{-(t-1) / 2} \leq C\left(y_{1} \cdots y_{S}\right)^{1 / 2}\left\|\nabla_{x} u\right\|_{L^{2}\left(\mathbb{R}^{n}\right)}^{1 / 2}\|u\|_{L^{2}\left(\mathbb{R}^{n}\right)}^{1 / 2}|x|^{-(t-1) / 2} .
$$

Due to the monotonicity-property in $y$-direction we also have $v(x) \geq y_{1} \cdots y_{s} u(x, y)$ and thus (2.9) gives the desired inequality.

We prove three additional lemmas which are used in the next section.

Lemma 6. The set $K_{t, s}$ is a weakly closed cone in $H^{1}\left(\mathbb{R}^{n}\right)$.

Proof. Take a sequence $\left(u_{k}\right)_{k \in \mathbb{N}} \subset K_{t, s}$ such that $u_{k} \rightarrow u \in H^{1}\left(\mathbb{R}^{n}\right)$ as $k \rightarrow \infty$. By the Sobolev embedding on bounded domains we deduce that a subsequence of $u_{k}$ converges pointwise almost everywhere on $\mathbb{R}^{n}$ to $u$. Since every $u_{k}$ enjoys the radial symmetry in the first component and the non-increasing property in the second variable, the pointwise convergence implies that also $u$ enjoys these properties, i.e., $u \in K_{t, s}$. 
Lemma 7. The functionals

$$
I(v)=\int_{\Omega} \frac{1}{2 r^{2}} F\left(r, z, r^{2} v^{2}\right) r^{3} d(r, z), \quad I^{\prime}(v)[v]=\int_{\Omega} f\left(r, z, r^{2} v^{2}\right) v^{2} r^{3} d(r, z)
$$

are weakly sequentially continuous on the set $K_{4,1} \subset H_{\text {cyl }}^{1}\left(r^{3} d r d z\right)$.

Remark: In the proof we use twice the following principle: if $S \subset \mathbb{R}^{m}$ is a set of finite measure and $w_{k}: S \rightarrow \mathbb{R}$ a sequence of measurable functions such that $\left\|w_{k}\right\|_{L^{r}(S)} \leq C$ and $w_{k} \rightarrow w$ pointwise a.e. as $k \rightarrow \infty$ then $\left\|w_{k}-w\right\|_{L^{q}(S)} \rightarrow 0$ as $k \rightarrow \infty$ for $1 \leq q<r$. The proof is as follows: Egorov's theorem allows to choose $\Sigma \subset S$ such that $w_{k} \rightarrow w$ uniformly on $\Sigma$ and $|S \backslash \Sigma| \leq \epsilon$ arbitrary small. By Hölder's inequality the remaining integral is estimated by $\int_{S \backslash \Sigma}\left|w_{k}-w\right|^{q} d x \leq \epsilon^{1-\frac{q}{r}}\left\|w_{k}-w\right\|_{L^{r}(S)}^{q}$.

Proof. Let us take a weakly convergent sequence $\left(v_{k}\right)_{k \in \mathbb{N}}$ in $K_{4,1}$ such that $v_{k} \rightarrow v$ in $H_{\text {cyl }}^{1}\left(r^{3} d r d z\right)$ and $v_{k} \rightarrow v$ pointwise a.e. in $\Omega$. By Lemma 6 one gets $v \in K_{4,1}$ and using Lemma 5 there exists a constant $C>0$ such that

$$
0 \leq v_{k}(r, z), v(r, z) \leq C r^{-\frac{3}{2}}|z|^{-\frac{1}{2}} \text { for all } k \in \mathbb{N} \text { and almost all }(r, z) \in \Omega .
$$

Our goal is now to show at least for a subsequence

$$
\int_{\Omega} \frac{1}{r^{2}} F\left(r, z, r^{2} v_{k}^{2}\right) r^{3} d(r, z) \rightarrow \int_{\Omega} \frac{1}{r^{2}} F\left(r, z, r^{2} v^{2}\right) r^{3} d(r, z) \text { as } k \rightarrow \infty
$$

and

$$
\int_{\Omega} f\left(r, z, r^{2} v_{k}^{2}\right) v_{k}^{2} r^{3} d(r, z) \rightarrow \int_{\Omega} f\left(r, z, r^{2} v^{2}\right) v^{2} r^{3} d(r, z) \text { as } k \rightarrow \infty .
$$

By (2.6) we find

$$
\frac{1}{r^{2}}\left|F\left(r, z, r^{2} v_{k}^{2}\right)-F\left(r, z, r^{2} v^{2}\right)\right| r^{3} \leq \epsilon r^{2}\left(v_{k}^{2}+v^{2}\right) r+C_{\epsilon}\left(\left|r v_{k}\right|^{p+1}+|r v|^{p+1}\right) r
$$

and hence

$$
\left(\left|F\left(r, z, r^{2} v_{k}^{2}\right)-F\left(r, z, r^{2} v^{2}\right)\right|-\epsilon r^{2}\left(v_{k}^{2}+v^{2}\right)\right)^{+} r \leq C_{\epsilon}\left(\left|r v_{k}\right|^{p+1}+|r v|^{p+1}\right) r .
$$

Inspired by [11] and [12] the idea is to show

$$
r v_{k} \rightarrow r v \text { in } L^{p+1}(r d r d z) \text { as } k \rightarrow \infty .
$$

Once (2.14) is established we obtain a majorant $\left|r v_{k}\right|,|r v| \leq w \in L^{p+1}(r d r d z)$ (cf. Lemma A.1 in [19]). Together with (2.13) this majorant allows to apply Lebesgue's dominated convergence theorem and yields

$$
\lim _{k \rightarrow \infty} \int_{\Omega}\left(\left|F\left(r, z, r^{2} v_{k}^{2}\right)-F\left(r, z, r^{2} v^{2}\right)\right|-\epsilon r^{2}\left(v_{k}^{2}+v^{2}\right)\right)^{+} r d r d z=2 \epsilon\|v\|_{L^{2}\left(r^{3} d r d z\right)}^{2}
$$

If we set

and

$$
a_{k}:=\int_{\Omega}\left|F\left(r, z, r^{2} v_{k}^{2}\right)-F\left(r, z, r^{2} v^{2}\right)\right| r d r d z
$$

then

$$
b_{k}:=\epsilon\left\|r^{2}\left(v_{k}^{2}+v^{2}\right)\right\|_{L^{1}(r d r d z)}=\epsilon\left(\left\|v_{k}\right\|_{L^{2}\left(r^{3} d r d z\right)}^{2}+\|v\|_{L^{2}\left(r^{3} d r d z\right)}^{2}\right) \leq C \epsilon
$$

$$
\begin{aligned}
\limsup _{k \in \mathbb{N}} a_{k} & \leq \limsup _{k \in \mathbb{N}} b_{k}+\limsup _{k \in \mathbb{N}}\left(a_{k}-b_{k}\right)^{+} \\
& \leq C \epsilon+\limsup _{k \in \mathbb{N}}\left(\int_{\Omega}\left(\left|F\left(r, z, r^{2} v_{k}^{2}\right)-F\left(r, z, r^{2} v^{2}\right)\right|-\epsilon r^{2}\left(v_{k}^{2}+v^{2}\right)\right) r d r d z\right)^{+}
\end{aligned}
$$




$$
\begin{aligned}
& \leq C \epsilon+\limsup _{k \in \mathbb{N}} \int_{\Omega}\left(\left|F\left(r, z, r^{2} v_{k}^{2}\right)-F\left(r, z, r^{2} v^{2}\right)\right|-\epsilon r^{2}\left(v_{k}^{2}+v^{2}\right)\right)^{+} r d r d z \\
& \leq \epsilon\left(C+2\|v\|_{L^{2}\left(r^{3} d r d z\right)}^{2}\right) \text { by (2.15). }
\end{aligned}
$$

Since $\epsilon>0$ was arbitrary this shows that $\lim _{k \rightarrow \infty} a_{k}=0$ and therefore (2.11) holds. The proof of (2.12) is similar since $\left(f\left(r, z, r^{2} v_{k}^{2}\right) r^{2} v_{k}^{2}-f\left(r, z, r^{2} v^{2}\right) r^{2} v^{2}-\epsilon r^{2}\left(v_{k}^{2}+v^{2}\right)\right)^{+} r$ satisfies an estimate just like (2.13) if we use (2.5) instead of (2.6).

It remains to prove (2.14). For this, we split our domain $\Omega$ into four parts $\Omega_{1}, \ldots, \Omega_{4}$ and show (2.14) on each of these parts separately. The definitions of $\Omega_{1}, \ldots, \Omega_{4}$ are as follows: For $R>0$ let

$$
\begin{array}{ll}
\Omega_{1}:=\{(r, z) \in \Omega: r<R,|z|<R\}, & \Omega_{2}:=\{(r, z) \in \Omega: r \geq R,|z| \geq R\}, \\
\Omega_{3}:=\{(r, z) \in \Omega: r<R,|z| \geq R\}, & \Omega_{4}:=\{(r, z) \in \Omega: r \geq R,|z|<R\} .
\end{array}
$$

Convergence on $\Omega_{1}$ : Follows from $r v_{k} \rightarrow r v$ in $L^{q}(K ; r d r d z)$ for every compact subset $K \subset[0, \infty) \times$ $\mathbb{R}$ and every $q \in[1,6)$. This step works independently of the choice of $R>0$.

Convergence on $\Omega_{2}$ : Let $\varepsilon>0$. With the help of (2.10) we calculate

$$
\begin{aligned}
\int_{\Omega_{2}}\left|r v_{k}-r v\right|^{p+1} r d(r, z) & \leq 2^{p+1} \int_{\Omega_{2}} r^{p+1}\left(\left|v_{k}\right|^{p+1}+|v|^{p+1}\right) r d(r, z) \\
& \leq 2^{p+1} C^{p-1} \int_{\Omega_{2}} r^{-\frac{p-1}{2}}|z|^{-\frac{p-1}{2}}\left(\left|v_{k}(r, z)\right|^{2}+|v(r, z)|^{2}\right) r^{3} d(r, z) \\
& \leq C_{1}\left(\left\|v_{k}\right\|_{H_{\mathrm{cyl}}^{1}\left(r^{3} d r d z\right)}^{2}+\|v\|_{H_{\mathrm{cyl}}^{1}\left(r^{3} d r d z\right)}^{2}\right) R^{-(p-1)} \leq C_{2} R^{-(p-1)}
\end{aligned}
$$

which is less or equal $\varepsilon$ if we choose $R>0$ large enough.

Convergence on $\Omega_{3}$ : Due to symmetry in $z$-direction it is enough to focus on $\tilde{\Omega}_{3}:=\{(r, z) \in \Omega: r<$ $R, z \geq R$. Let $\alpha>0$ be arbitrary. Again by (2.10) we obtain

$$
\left\{(r, z) \in \tilde{\Omega}_{3}: v_{k}(r, z)>\alpha\right\} \subset\left\{(r, z) \in \tilde{\Omega}_{3}: r z^{\frac{1}{3}} \leq C_{\alpha}\right\}=: S_{\alpha},
$$

where $C_{\alpha}=(C / \alpha)^{2 / 3}$ and $C$ is the constant from (2.10). The set $S_{\alpha}$ has finite measure since

$$
\left|S_{\alpha}\right| \leq \int_{R}^{\infty} \int_{0}^{C_{\alpha} z^{-1 / 3}} r^{3} d r d z=\frac{C_{\alpha}^{4}}{4} \int_{R}^{\infty} z^{-\frac{4}{3}} d z=\frac{3}{4} C_{\alpha}^{4} R^{-\frac{1}{3}}<\infty .
$$

By the convergence principle from the remark above and since by (2.3) $\left\|r v_{k}\right\|_{L^{6}(r d r d z)} \leq\left\|v_{k}\right\|_{H_{\mathrm{cyl}}^{1}\left(r^{3} d r d z\right)}$ is bounded we obtain $\int_{S_{\alpha}} r^{p-1}\left|v_{k}-v\right|^{p+1} r^{3} d(r, z) \rightarrow 0$ as $k \rightarrow \infty$ for $1 \leq p<5$. It remains to prove the convergence on $\tilde{\Omega}_{3} \backslash S_{\alpha}$. For allmost all $(r, z) \in \tilde{\Omega}_{3} \backslash S_{\alpha}$ we have that $v(r, z)=\lim _{k \rightarrow \infty} v_{k}(r, z) \leq \alpha$. Hence,

$$
\int_{\tilde{\Omega}_{3} \backslash S_{\alpha}} r^{p-1}\left|v_{k}-v\right|^{p+1} r^{3} d(r, z) \leq R^{p-1}(2 \alpha)^{p-1} \int_{\Omega}\left|v_{k}-v\right|^{2} r^{3} d(r, z) \leq C \alpha^{p-1} .
$$

In summary, since $\alpha>0$ is arbitrary this shows 2.14 on $\Omega_{3}$.

Convergence on $\Omega_{4}$ : Again it is enough to focus on $\tilde{\Omega}_{4}:=\{(r, z) \in \Omega: r \geq R, 0 \leq z<R\}$. Fix $z \in(0, R)$. Let us first show that

$$
\int_{\{r \geq R\}} r^{p-1}\left|v_{k}(r, z)-v(r, z)\right|^{p+1} r^{3} d r \rightarrow 0 \text { as } k \rightarrow \infty .
$$


Since $v_{k}(r, \cdot)$ is nonincreasing in its last component we deduce

$$
\int_{0}^{\infty} r^{q} v_{k}^{q}(r, z) r d r \leq \frac{1}{z} \int_{0}^{z} \int_{0}^{\infty} r^{q} v_{k}^{q}(r, \zeta) r d r d \zeta \leq \frac{1}{z} \int_{\Omega} r^{q} v_{k}^{q}(r, \zeta) r d(r, \zeta) \leq \frac{C}{z}
$$

for all $q \in[2,6]$ by (2.3). Thus for $q \in[2,6]$ the sequence $\left\|\cdot v_{k}(\cdot, z)\right\|_{L^{q}((0, \infty), r d r)}$ is uniformly bounded in $k \in \mathbb{N}$. Moreover, (2.10) implies $v_{k}(r, z) \leq C(z) r^{-\frac{3}{2}}$ uniformly in $k \in \mathbb{N}$. Hence for $\tilde{R}>R$

$$
\begin{aligned}
\int_{\tilde{R}}^{\infty} r^{p-1}\left|v_{k}(r, z)-v(r, z)\right|^{p+1} r^{3} d r & \leq(2 C(z))^{p-1} \int_{\tilde{R}}^{\infty} r^{-\frac{p-1}{2}}\left|v_{k}(r, z)-v(r, z)\right|^{2} r^{3} d r \\
& \leq(2 C(z))^{p-1} \tilde{R}^{\frac{1-p}{2}} \frac{C}{z} \text { by (2.17). }
\end{aligned}
$$

The last term can be made arbitrarily small provided $\tilde{R}$ is chosen big enough. To finish the proof of (2.16) it remains to prove $\int_{R}^{\tilde{R}} r^{p-1}\left|v_{k}(r, z)-v(r, z)\right|^{p+1} r^{3} d r \rightarrow 0$ as $k \rightarrow \infty$. Since for almost all $z \in(0, R)$ we have $v_{k}(\cdot, z) \rightarrow v(\cdot, z)$ pointwise almost everywhere on $(R, \tilde{R})$ as well as the boundedness of $\left\|\cdot v_{k}(\cdot, z)\right\|_{L^{6}((0, \infty), r d r)}$ by (2.17) we can apply the convergence principle from the remark above and deduce

$$
\int_{R}^{\tilde{R}} r^{p-1}\left|v_{k}(r, z)-v(r, z)\right|^{p+1} r^{3} d r \rightarrow 0 \text { as } k \rightarrow \infty .
$$

Hence (2.16) is accomplished for almost all $z \in(0, R)$.

Defining $\varphi_{k}(z):=\int_{\{r \geq R\}} r^{p-1}\left|v_{k}(r, z)-v(r, z)\right|^{p+1} r^{3} d r$ we have $\varphi_{k} \rightarrow 0$ as $k \rightarrow \infty$ pointwise almost everywhere in $[0, R)$. The sequence $\left(\varphi_{k}\right)_{k \in \mathbb{N}}$ is bounded in $L^{1}([0, R), d z)$ since by (2.2)

$$
\int_{0}^{R} \int_{\{r \geq R\}} r^{p-1}\left|v_{k}(r, z)-v(r, z)\right|^{p+1} r^{3} d r d z \leq C \int_{\Omega} r^{p-1}\left(\left|v_{k}\right|^{p+1}+|v|^{p+1}\right) r^{3} d(r, z) \leq \tilde{C} .
$$

Moreover, for $p \in(1,3]$, the sequence $\left(\varphi_{k}\right)_{k \in \mathbb{N}}$ is bounded in $W^{1,1}([0, R), d z)$ since

$$
\begin{aligned}
\left\|\frac{\partial \varphi_{k}}{\partial z}\right\|_{L^{1}([0, R], d z)}^{2} & \leq\left(\int_{0}^{R} \int_{R}^{\infty}(p+1) r^{p-1}\left|v_{k}-v\right|^{p}\left|\frac{\partial v_{k}}{\partial z}-\frac{\partial v}{\partial z}\right| r^{3} d r d z\right)^{2} \\
& \leq\left(\int_{\Omega}(p+1) r^{p-1}\left|v_{k}-v\right|^{p}\left|\frac{\partial v_{k}}{\partial z}-\frac{\partial v}{\partial z}\right| r^{3} d(r, z)\right)^{2} \\
& \leq C \int_{\Omega} r^{2 p-2}\left|v_{k}-v\right|^{2 p} r^{3} d(r, z) \int_{\Omega}\left|\frac{\partial v_{k}}{\partial z}-\frac{\partial v}{\partial z}\right|^{2} r^{3} d(r, z) \\
& =C\left\|r\left(v_{k}-v\right)\right\|_{L^{2 p}(r d r d z)}^{2 p} \int_{\Omega}\left|\frac{\partial v_{k}}{\partial z}-\frac{\partial v}{\partial z}\right|^{2} r^{3} d(r, z) \leq C .
\end{aligned}
$$

Hence, by the compact embedding $W^{1,1}([0, R), d z) \hookrightarrow L^{1}([0, R), d z)$ we conclude that at least a subsequence of $\left(\varphi_{k}\right)_{k \in \mathbb{N}}$ is converging in $L^{1}([0, R), d z)$ to a limit function, which must be 0 since we have already asserted the pointwise a.e. convergence to 0 on $[0, R)$. This shows (2.14) on $\Omega_{4}$ for $p \in(1,3]$. For $p \in(3,5)$ we make use of Hölder's interpolation, namely,

$$
\left\|r v_{k}-r v\right\|_{L_{\mathrm{cyl}}^{p+1}\left(\Omega_{4}, r d r d z\right)}^{p+1} \leq\left\|r v_{k}-r v\right\|_{L_{\mathrm{cyl}}^{4}\left(\Omega_{4}, r d r d z\right)}^{4 \theta}\left\|r v_{k}-r v\right\|_{L_{\mathrm{cyl}}^{6}\left(\Omega_{4}, r d r d z\right)}^{6(1-\theta)} \leq \tilde{C}\left\|r v_{k}-r v\right\|_{L_{\mathrm{cyl}}^{4}\left(\Omega_{4}, r d r d z\right)}^{4 \theta} \rightarrow 0
$$

as $k \rightarrow \infty$, where $\theta \in(0,1)$ is chosen such that $p+1=4 \theta+6(1-\theta)$, i.e., $\theta=\frac{5-p}{2}$.

The combination of convergences on $\Omega_{1}, \ldots, \Omega_{4}$ finally proves (2.14).

For our last lemma we need the notion of cylindrical $C_{c}^{\infty}$-functions which we introduce now. 
Definition 8. A function $u=u(r, z)$ belongs to $C_{c}^{\infty}([0, \infty) \times \mathbb{R})$ if and only if $u \in C^{\infty}([0, \infty) \times \mathbb{R})$, $\operatorname{supp} u$ is compact in $[0, \infty) \times \mathbb{R}$ and $\frac{\partial^{j} u}{\partial r^{j}}(0, z)=0$ for all odd integers $j \in 2 \mathbb{N}-1$.

Remark: Since $u \in C_{c}^{\infty}([0, \infty) \times \mathbb{R})$ is equivalent to $\tilde{u} \in C_{c}^{\infty}\left(\mathbb{R}^{5}\right)$ with $\tilde{u}(x):=u\left(\left|\left(x_{1}, \ldots, x_{4}\right)\right|, x_{5}\right)$ we see that $C_{c}^{\infty}([0, \infty) \times \mathbb{R})$ is dense in $H_{\text {cyl }}^{1}\left(r^{3} d r d z\right)$.

Lemma 9. For $u \in H_{\mathrm{cyl}}^{1}\left(r^{3} d r d z\right)$ we have $\left\|u^{\star}\right\| \leq\|u\|$ where $\star$ denotes Steiner-symmetrization with respect to $z$ and $\|\cdot\|$ is the equivalent norm from Theorem 11 Moreover

$$
I(u) \leq I\left(u^{\star}\right) \quad \text { and } \quad I^{\prime}(u)[u] \leq I^{\prime}\left(u^{\star}\right)\left[u^{\star}\right] .
$$

Proof. We begin by recalling several classical rearrangement inequalities from [9], [10]. Recall first the Pólya-Szegö inequality

$$
\int_{\mathbb{R}^{n}}\left|\nabla f^{\circledast}\right|^{2} d x \leq \int_{\mathbb{R}^{n}}|\nabla f|^{2} d x
$$

for $f \in H^{1}\left(\mathbb{R}^{n}\right)$ and ${ }^{\circledast}$ denoting Schwarz-symmetrization (also called symmetrically decreasing rearrangement). Furthermore we have for $0 \leq f, g \in L^{2}\left(\mathbb{R}^{n}\right)$ the classical rearrangement inequality

$$
\int_{\mathbb{R}} f g d x \leq \int_{\mathbb{R}} f^{\circledast} g^{\circledast} d x
$$

and the nonexpansivity of rearrangement

$$
\int_{\mathbb{R}^{n}}\left|f^{\circledast}-g^{\circledast}\right|^{2} d x \leq \int_{\mathbb{R}^{n}}|f-g|^{2} d x .
$$

From (2.18) we immediately receive for $u \in H_{\text {cyl }}^{1}\left(r^{3} d r d z\right)$ that

$$
\int_{\mathbb{R}}\left|\nabla_{z} u^{\star}\right|^{2} d z \leq \int_{\mathbb{R}}\left|\nabla_{z} u\right|^{2} d z
$$

Next we want to establish a similar inequality for $\nabla_{r} u$. We do this first for $u \in C_{c}^{\infty}([0, \infty) \times \mathbb{R})$. With the help of (2.20) we find that

$$
\int_{\mathbb{R}}\left|\frac{u^{\star}(r+t, z)-u^{\star}(r, z)}{t}\right|^{2} d z \leq \int_{\mathbb{R}}\left|\frac{u(r+t, z)-u(r, z)}{t}\right|^{2} d z
$$

for almost all $r, t \in[0, \infty)$. Sending $t \rightarrow 0$ and using Fatou's lemma on the left side of the inequality yields

$$
\int_{\mathbb{R}}\left|\nabla_{r} u^{\star}\right|^{2} d z \leq \int_{\mathbb{R}}\left|\nabla_{r} u\right|^{2} d z
$$

for $u \in C_{c}^{\infty}([0, \infty) \times \mathbb{R})$ and almost all $r \in[0, \infty)$. Since Steiner Symmetrization is continuous in $H^{1}$ (see Theorem 1 in [7]) we obtain by approximation that (2.22) is indeed valid for all $u \in H_{c y l}^{1}\left(r^{3} d r d z\right)$. Together with (2.21) we obtain $\int_{\mathbb{R}}\left|\nabla_{r, z} u^{\star}\right|^{2} d z \leq \int_{\mathbb{R}}\left|\nabla_{r, z} u\right|^{2} d z$ for almost all $r \geq 0$ and integration leads to

$$
\int_{\mathbb{R}} \int_{0}^{\infty}\left|\nabla_{r, z} u^{\star}\right|^{2} r^{3} d r d z \leq \int_{\mathbb{R}} \int_{0}^{\infty}\left|\nabla_{r, z} u\right|^{2} r^{3} d r d z .
$$

Fixing $r \in[0, \infty)$ and applying (2.19) to $f(\cdot)=\operatorname{ess} \sup V-V(r, \cdot)$ and $g(\cdot)=u^{2}(r, \cdot)$ gives

$$
\begin{aligned}
\int_{\mathbb{R}}(\operatorname{ess} \sup V-V(r, \cdot)) u^{2}(r, \cdot) d z & \leq \int_{\mathbb{R}}(\operatorname{ess} \sup V-V(r, \cdot))^{\star}\left(u^{2}\right)^{\star}(r, \cdot) d z \\
& =\int_{\mathbb{R}}(\operatorname{ess} \sup V-V(r, \cdot))\left(u^{\star}\right)^{2}(r, \cdot) d z .
\end{aligned}
$$


Using $\|u(r, \cdot)\|_{L^{2}(\mathbb{R})}=\left\|u^{\star}(r, \cdot)\right\|_{L^{2}(\mathbb{R})}$ this results in

$$
\int_{\mathbb{R}} \int_{0}^{\infty} V(r, z)\left(u^{\star}\right)^{2} r^{3} d r d z \leq \int_{\mathbb{R}} \int_{0}^{\infty} V(r, z) u^{2} r^{3} d r d z .
$$

The combination of (2.23) and (2.24) yields the claimed inequality $\left\|u^{\star}\right\|^{2} \leq\|u\|^{2}$.

Assumption (v) on $f$ allows to apply Theorem 5.1 in [6] and to deduce

$$
I^{\prime}(u)[u]=\int_{\Omega} f\left(r, z, r^{2} u^{2}\right) u^{2} r^{3} d(r, z) \leq \int_{\Omega} f\left(r, z, r^{2} u^{\star 2}\right) u^{\star 2} r^{3} d(r, z)=I^{\prime}\left(u^{\star}\right)\left[u^{\star}\right] .
$$

Moroever, using (v) with $s=0$ shows that for all $r \in[0, \infty), \sigma \geq 0$ the function $z \mapsto f\left(r, z, \sigma^{2}\right)$ is symmetrically nonincreasing in $z$ and hence

$$
\Phi_{\sigma}(r, z, s):=F\left(r, z, r^{2}(s+\sigma)^{2}\right)-F\left(r, z, r^{2} s^{2}\right)=\int_{r^{2} s^{2}}^{r^{2}(s+\sigma)^{2}} f(r, z, t) d t
$$

is symmetrically nonincreasing in $z$. Applying once more Theorem 5.1 in [6] yields

$$
I(u)=\int_{\Omega} \frac{1}{2 r^{2}} F\left(r, z, r^{2} u^{2}\right) r^{3} d(r, z) \leq \int_{\Omega} \frac{1}{2 r^{2}} F\left(r, z, r^{2} u^{\star 2}\right) r^{3} d(r, z)=I\left(u^{\star}\right) .
$$

This finishes the proof of the lemma.

\section{Proof of Theorem 1}

Proof. Recall from Lemma 7 the definition $I(u):=\int_{\Omega} \frac{1}{2 r^{2}} F\left(r, z, r^{2} u^{2}\right) r^{3} d(r, z)$ for $u \in H_{\text {cyl }}^{1}\left(r^{3} d r d z\right)$. We show that the assumptions (i)-(iii) of Theorem 12 in [18] are satisfied. Let $\varepsilon>0$. The growth assumptions (i) and (ii) on $f$ imply that for every $\epsilon>0$ there exists $C_{\epsilon}>0$ such that the global estimate $0 \leq f(r, z, s) \leq \epsilon+C_{\epsilon}|s|^{\frac{p-1}{2}}$ holds. Together with (2.2) we obtain

$$
\begin{aligned}
\left|I^{\prime}(u)[v]\right| & =\left|\int_{\Omega} f\left(r, z, r^{2} u^{2}\right) u v r^{3} d(r, z)\right| \\
& \leq \varepsilon \int_{\Omega}|r u \| r v| r d(r, z)+C_{\epsilon} \int_{\Omega}|r u|^{p}|r v| r d(r, z) \\
& \leq \varepsilon C\|u\|_{H_{c y l}^{1}\left(r^{3} d r d z\right)}\|v\|_{H_{c y l}^{1}\left(r^{3} d r d z\right)}+\tilde{C}_{\epsilon}\|u\|_{H_{c y l}^{1}\left(r^{3} d r d z\right)}^{p}\|v\|_{H_{c y l}^{1}\left(r^{3} d r d z\right)}
\end{aligned}
$$

Taking the supremum over all $v \in H_{c y l}^{1}\left(r^{3} d r d z\right)$ with $\|v\|_{H_{c y l}^{1}\left(r^{3} d r d z\right)}=1$ we see that

$$
I^{\prime}(u)=o(\|u\|) \text { as } u \rightarrow 0 .
$$

Moreover, due to assumption (iii) on $f$ the map

$$
s \mapsto \frac{I^{\prime}(s u)[u]}{s}=\int_{\Omega} f\left(r, z, s^{2} r^{2} u^{2}\right) u^{2} r^{3} d(r, z) \text { is strictly increasing for all } u \neq 0 \text { and } s>0 .
$$

Next we claim that

$$
\frac{I(s u)}{s^{2}} \rightarrow \infty \text { as } s \rightarrow \infty \text { uniformly for } u \text { on weakly compact subsets } W \text { of } H_{c y l}^{1}\left(r^{3} d r d z\right) \backslash\{0\} .
$$

Suppose not. Then there are $\left(u_{k}\right)_{k \in \mathbb{N}} \subset W$ and $s_{k} \rightarrow \infty$ as $k \rightarrow \infty$ such that $\frac{I\left(s_{k} u_{k}\right)}{s_{k}^{2}}$ is bounded as $k \rightarrow \infty$. But along a subsequence we have $u_{k} \rightarrow u \neq 0$ and $u_{k}(x) \rightarrow u(x)$ pointwise almost everywhere. Let 
$\Omega^{\sharp}:=\{(r, z) \in \Omega: u(r, z) \neq 0\}$. Then $\left|\Omega^{\sharp}\right|>0$ and on $\Omega^{\sharp}$ we have $\left|s_{k} u_{k}(r, z)\right| \rightarrow \infty$ as $k \rightarrow \infty$. Fatou's lemma and assumption (iv) on $F$ imply

$$
\frac{I\left(s_{k} u_{k}\right)}{s_{k}^{2}}=\int_{\Omega} \frac{F\left(r, z, s_{k}^{2} r^{2} u_{k}^{2}\right)}{2 s_{k}^{2} r^{2}} r^{3} d(r, z) \geq \int_{\Omega^{\sharp}} \frac{F\left(r, z, s_{k}^{2} r^{2} u_{k}^{2}\right)}{2 s_{k}^{2} r^{2} u_{k}^{2}} u_{k}^{2} r^{3} d(r, z) \rightarrow \infty \text { as } k \rightarrow \infty,
$$

a contradiction. In summary, (3.1), (3.2), (3.3) imply that (i)-(iii) of Theorem 12 in [18] are satisfied.

Now we take a sequence $\left(u_{k}\right)_{k \in \mathbb{N}} \subset M$ such that $J\left(u_{k}\right) \rightarrow \inf _{M} J$ as $k \rightarrow \infty$. Since $\left\|\nabla_{r, z}\left|u_{k}\right|\right\|_{L^{2}}=$ $\left\|\nabla_{r, z} u_{k}\right\|_{L^{2}}$ we can assume that $u_{k} \geq 0$ for all $k \in \mathbb{N}$. Then Theorem 12 in [18] guarantees that for every $k$ there is a unique $t_{k}>0$ such that $v_{k}:=t_{k} u_{k}^{\star} \in M$. We show next that $t_{k} \leq 1$ for all $k \in \mathbb{N}$. Assume $t_{k}>1$. Then

$$
\begin{aligned}
\int_{\Omega} f\left(r, z, r^{2} u_{k}^{\star 2}\right) u_{k}^{\star 2} r^{3} d(r, z) & <\int_{\Omega} f\left(r, z, t_{k}^{2} r^{2} u_{k}^{\star 2}\right) u_{k}^{\star 2} r^{3} d(r, z) \quad \text { by assumption (iii) } \\
& =\left\|u_{k}^{\star}\right\|^{2} \quad \text { since } t_{k} u_{k}^{\star} \in M \\
& \leq\left\|u_{k}\right\|^{2} \quad \text { by Lemma } 9 \\
& =\int_{\Omega} f\left(r, z, r^{2} u_{k}^{2}\right) u_{k}^{2} r^{3} d(r, z) \quad \text { since } u_{k} \in M .
\end{aligned}
$$

This contradicts the inequality $I^{\prime}\left(u_{k}\right)\left[u_{k}\right] \leq I^{\prime}\left(u_{k}^{\star}\right)\left[u_{k}^{\star}\right]$ from Lemma 9 and thus $t_{k} \leq 1$ for all $k \in \mathbb{N}$.

Next notice that for fixed $(r, z, s) \in[0, \infty) \times \mathbb{R} \times[0, \infty)$ and $t \in(0,1]$ one has

$$
\frac{d}{d t}\left(t^{2} f\left(r, z, s^{2}\right) s^{2}-F\left(r, z, t^{2} s^{2}\right)\right)=2 t s^{2}\left(f\left(r, z, s^{2}\right)-f\left(r, z, t^{2} s^{2}\right)\right)>0
$$

since $f$ is strictly increasing in its last variable by assumption (iii). This shows that the map $t \mapsto$ $t^{2} f\left(r, z, s^{2}\right) s^{2}-F\left(r, z, t^{2} s^{2}\right)$ is strictly increasing for $t \in[0,1]$. From this monotonicity and the inequality $I\left(t_{k} u_{k}\right) \leq I\left(t_{k} u_{k}^{\star}\right)$ from Lemma 9 we conclude

$$
\begin{aligned}
2 J\left(v_{k}\right) & =\int_{\Omega}\left(t_{k}^{2}\left|\nabla_{r, z} u_{k}^{\star}\right|^{2}+V(r, z) t_{k}^{2} u_{k}^{\star 2}-\frac{1}{r^{2}} F\left(r, z, r^{2} t_{k}^{2} u_{k}^{\star 2}\right)\right) r^{3} d(r, z) \\
& \leq \int_{\Omega}\left(t_{k}^{2}\left|\nabla_{r, z} u_{k}\right|^{2}+V(r, z) t_{k}^{2} u_{k}^{2}-\frac{1}{r^{2}} F\left(r, z, r^{2} t_{k}^{2} u_{k}^{2}\right)\right) r^{3} d(r, z) \\
& =\int_{\Omega} \frac{1}{r^{2}}\left(f\left(r, z, r^{2} u_{k}^{2}\right) t_{k}^{2} r^{2} u_{k}^{2}-F\left(r, z, r^{2} t_{k}^{2} u_{k}^{2}\right)\right) r^{3} d(r, z) \\
& \leq \int_{\Omega} \frac{1}{r^{2}}\left(f\left(r, z, r^{2} u_{k}^{2}\right) r^{2} u_{k}^{2}-F\left(r, z, r^{2} u_{k}^{2}\right)\right) r^{3} d(r, z) \\
& =2 J\left(u_{k}\right) .
\end{aligned}
$$

So $\left(v_{k}\right)_{k \in \mathbb{N}} \subset M$ is also a minimizing sequence for $J$ which belongs to $K_{4,1}$. The boundedness of $\left(v_{k}\right)_{k \in \mathbb{N}}$ is established in Proposition 14 in [18]. Hence, we find $v_{\infty} \in H_{\text {cyl }}^{1}\left(r^{3} d r d z\right)$ such that $v_{k} \rightarrow v_{\infty}$ in $H_{\text {cyl }}^{1}\left(r^{3} d r d z\right)$ along a subsequence as $k \rightarrow \infty$. In addition, $v_{\infty} \in K_{4,1}$ due to Lemma 6 and $v_{\infty} \neq 0$ by Proposition 14 in [18] where instead of the weak sequential continuity of $I$ on all of $H_{\text {cyl }}^{1}\left(r^{3} d r d z\right)$ we use it only on $K_{4,1}$ as stated in Lemma7

Let us show that $v_{\infty} \in M$. Since $v_{\infty} \neq 0$ we can choose $t_{\infty}>0$ such that $t_{\infty} v_{\infty} \in M$. In the same manner as before for the sequence $t_{k}$ we can show that $t_{\infty} \leq 1$. Assume $t_{\infty}<1$. Then as in (3.4) and using the weak sequential continuity on $K_{4,1}$ as shown in Lemma 7 we find

$$
2 J\left(t_{\infty} v_{\infty}\right)<\int_{\Omega} \frac{1}{r^{2}}\left(f\left(r, z, r^{2} v_{\infty}^{2}\right) r^{2} v_{\infty}^{2}-F\left(r, z, r^{2} v_{\infty}^{2}\right)\right) r^{3} d(r, z)
$$




$$
\begin{aligned}
& =\lim _{k \rightarrow \infty} \int_{\Omega} \frac{1}{r^{2}}\left(f\left(r, z, r^{2} v_{k}^{2}\right) r^{2} v_{k}^{2}-F\left(r, z, r^{2} v_{k}^{2}\right)\right) r^{3} d(r, z) \\
& =2 \inf _{M} J \leq 2 J\left(t_{\infty} v_{\infty}\right)
\end{aligned}
$$

which is a contradiction. So $t_{\infty}=1$ and thus $v_{\infty} \in M$. Then by the weak lower semi-continuity of $\|\cdot\|$ and once again the weak sequential continuity of $I$ we conclude

$$
J\left(v_{\infty}\right) \leq \liminf _{k \rightarrow \infty} J\left(v_{k}\right)=\inf _{M} J \leq J\left(v_{\infty}\right) .
$$

Hence, $v_{\infty} \in K_{4,1}$ is a minimizer of $J$ on $M$, i.e., a ground state of (1.3) which is Steiner symmetric in $z$ with respect to $\{z=0\}$.

\section{APPENDIX}

Here we prove that the condition $V \geq 0$ and $\inf _{B_{R}^{c}} V>0$ for some $R>0$ implies that on $H_{\text {cyl }}^{1}\left(r^{3} d r d z\right)$ the expression $\left(\int_{\Omega}\left(\left|\nabla_{r, z} u\right|^{2}+V(r, z) u^{2}\right) r^{3} d(r, z)\right)^{\frac{1}{2}}$ is an equivalent norm. Suppose not. Then there is a sequence $\left(u_{k}\right)_{k \in \mathbb{N}}$ such that $\left\|u_{k}\right\|_{L^{2}\left(r^{3} d r d z\right)}=1$ and $\int_{\Omega}\left(\left|\nabla_{r, z} u_{k}\right|^{2}+V(r, z) u_{k}^{2}\right) r^{3} d(r, z) \rightarrow 0$ as $k \rightarrow \infty$. In particular,

$$
\int_{\Omega}\left|\nabla_{r, z} u_{k}\right|^{2} r^{3} d(r, z) \rightarrow 0 \text { and } \int_{B_{R}^{c}} u_{k}^{2} r^{3} d(r, z) \rightarrow 0 \text { as } k \rightarrow \infty .
$$

Let $\chi$ denote a smooth cut-off function such that $\chi(r, z)=1$ for $0 \leq \sqrt{r^{2}+z^{2}}<R$ and $\chi(r, z)=0$ for $\sqrt{r^{2}+z^{2}} \geq R+1$. Then $v_{k}:=\chi u_{k} \in H_{0, \mathrm{cyl}}^{1}\left(B_{R+1}, r^{3} d r d z\right)$ and

$$
\left|\nabla_{r, z} v_{k}\right|^{2}=\chi^{2}\left|\nabla_{r, z} u_{k}\right|^{2}+\left|\nabla_{r, z} \chi\right|^{2} u_{k}^{2}+2 u_{k} \chi \nabla_{r, z} u_{k} \cdot \nabla_{r, z} \chi
$$

Hence, by (3.5)

$$
\begin{aligned}
\int_{\Omega}\left|\nabla_{r, z} v_{k}\right|^{2} r^{3} d(r, z) & \leq 2 \int_{\Omega} \chi^{2}\left|\nabla_{r, z} u_{k}\right|^{2} r^{3} d(r, z)+2 \int_{\Omega} u_{k}^{2}\left|\nabla_{r, z} \chi\right|^{2} r^{3} d(r, z) \\
& \leq 2 \int_{\Omega}\left|\nabla_{r, z} u_{k}\right|^{2} r^{3} d(r, z)+2\left\|\nabla_{r, z} \chi\right\|_{\infty}^{2} \int_{B_{R+1} \backslash B_{R}} u_{k}^{2} r^{3} d(r, z) \rightarrow 0 \text { as } k \rightarrow \infty
\end{aligned}
$$

In particular, $\int_{B_{R+1}}\left|\nabla_{r, z} v_{k}\right|^{2} r^{3} d(r, z) \rightarrow 0$ as $k \rightarrow \infty$. By Poincaré's inequality, $\left\|u_{k}\right\|_{L^{2}\left(r^{3} d r d z\right)}=1$ and (3.5) we see

$$
C_{P} \int_{B_{R+1}}\left|\nabla_{r, z} v_{k}\right|^{2} r^{3} d(r, z) \geq \int_{B_{R+1}} v_{k}^{2} r^{3} d(r, z) \geq \int_{B_{R}} u_{k}^{2} r^{3} d(r, z)=1-o(1),
$$

contradicting (3.6).

\section{ACKNOWLEDGEMENT}

We gratefully acknowledge financial support by the Deutsche Forschungsgemeinschaft (DFG) through CRC 1173. 


\section{REFERENCES}

[1] Azzollini, A., Benci, V., D’Aprile, T. and Fortunato, D.: Existence of static solutions of the semilinear Maxwell equations. Ricerche di matematica, 55(2), 123-137, (2006).

[2] Bartsch, T., Dohnal, T., Plum, M. and Reichel, W.: Ground states of a nonlinear curl-curl problem in cylindrically symmetric media. arXiv preprint arXiv:1411.7153, (2014).

[3] Bartsch, T. and Mederski, J.: Ground and bound state solutions of semilinear time-harmonic Maxwell equations in a bounded domains. Arch. Ration. Mech. Anal., 215(1), 283-306, (2015).

[4] Bartsch, T. and Mederski, J.: Nonlinear time-harmonic Maxwell equations in an anisotropic bounded medium. arXiv preprint arXiv:1509.01994. (2015).

[5] Benci, V. and Fortunato, D.: Towards a unified field theory for classical electrodynamics. Arch. Ration. Mech. Anal., 173(3), 379-414, (2004).

[6] Brock, F.: Continuous rearrangement and symmetry of solutions of elliptic problems. Proceedings of the Indian Academy of Sciences-Mathematical Sciences. Vol. 110. No. 2. Springer India, (2000).

[7] Burchard, A.: Steiner symmetrization is continuous in $W^{1, p}$. Geometric \& Functional Analysis GAFA 7.5: 823860, (1997).

[8] D’Aprile, T. and Siciliano, G.: Magnetostatic solutions for a semilinear perturbation of the Maxwell equations. Adv. Differential Equations, 16(5-6), 435-466, (2011).

[9] Lieb, E. H.: Existence and uniqueness of the minimizing solution of Choquard's nonlinear equation. Studies in Applied Mathematics 57: 93-105, (1977).

[10] Lieb, E. H. and Loss, M.: Analysis, volume 14 of graduate studies in mathematics. American Mathematical Society, Providence, RI, 4, (2001).

[11] Lions, P.-L.: Minimization problems in $L^{1}\left(\mathbb{R}^{3}\right)$. Journal of Functional Analysis, 41(2), 236-275, (1981).

[12] Lions, P.-L.: Symétrie et compacité dans les espaces de Sobolev. Journal of Functional Analysis 49.3: 315-334, (1982).

[13] Liu, S.: On superlinear problems without the Ambrosetti and Rabinowitz condition. Nonlinear Analysis: Theory, Methods \& Applications, 73(3), 788-795, (2010).

[14] Mederski, J.: Ground states of time-harmonic semilinear Maxwell equations in $\mathbb{R}^{3}$ with vanishing permittivity. arXiv preprint arXiv:1406.4535, (2014), to appear in Arch. Ration. Mech. Anal.

[15] Nehari, Z.: On a class of nonlinear second-order differential equations. Transactions of the American Mathematical Society 95.1: 101-123, (1960).

[16] Nehari, Z.: Characteristic values associated with a class of nonlinear second-order differential equations. Acta Mathematica 105.3: 141-175, (1961).

[17] Stuart, C. A.: A variational approach to bifurcation in $L^{p}$ on an unbounded symmetrical domain. Mathematische Annalen, 263(1), 51-59, (1983).

[18] Szulkin, A. and Weth, T.: The method of Nehari manifold. Handbook of nonconvex analysis and applications: 597-632, (2010).

[19] Willem, M.: Minimax theorems. Vol. 24. Springer Science \& Business Media, (1997).

A. HIRSCH

Institute for AnAlysis, KarlsRuhe Institute of Technology (KIT),

D-76128 KarlsRuHe, Germany

E-mail address: andreas.hirsch@kit.edu

W. REICHEL

Institute for Analysis, Karlsruhe Institute of Technology (KIT),

D-76128 Karlsruhe, Germany

E-mail address: wolfgang.reichel@kit.edu 